\title{
Diffuse Optical Tomography
}

National Cancer Institute

\section{Source}

National Cancer Institute. Diffuse Optical Tomography. NCI Thesaurus. Code C116446.

A type of computer tomography that creates 3D, digital models of objects by constructing images created by interpreting light that has been transmitted and scattered through an object. Because the object being imaged must be at least partially translucent, this technique works best on soft tissues. 